\title{
Dipolar Order in Molecular Fluids: I. Toward an Understanding
}

\author{
Gunnar Karlström • Per Linse
}

Received: 31 March 2011 / Accepted: 8 September 2011 / Published online: 7 October 2011

(C) The Author(s) 2011. This article is published with open access at Springerlink.com

\begin{abstract}
The origin behind the dipolar order in molecular fluids is investigated by using a simple dipolar fluid and Monte Carlo simulation technique. A penalty function is employed to separately manipulate the positional and orientational structure of the fluid. By considering the distance-dependent Kirkwood function $G_{\mathrm{k}}$, which in turn is related to the dielectric permittivity of the fluid, it is observed that both positional and orientational ordering are involved to establish dipolar order.
\end{abstract}

Keywords Dipolar order · Molecular fluid · Understanding $\cdot$ Penalty function

\section{Introduction}

The first attempt to understand the dielectric behavior of molecular media dates back to the middle of the 19th century and the work of Clausius [1] and Mossotti [2]. They developed independently the well-known Clausius-Mossotti equation. Later, in the first half of the 20th century, Langevin [3], Onsager [4], and Kirkwood [5] made important contributions to our understanding of the dielectric behavior of a molecular fluid. In particular, we note that Kirkwood introduced the today well-known Kirkwood $g_{\mathrm{K}}$-factor to describe the dipolar correlations of relevance for the dielectric behavior of dipolar fluids. In the 70ies of the last century, Niehuis and Deutch [6] and somewhat later Wertheim [7] further developed our understanding of these systems.

First principle statistic mechanical modeling of the dielectric behavior of molecular fluids started roughly also at the 70ies and pioneering work [8-10], to mention a few, further increased our understanding of the systems. In the beginning of the 80ies the problem of

\footnotetext{
G. Karlström

Division of Theoretical Chemistry, Department of Chemistry, Lund University, P.O. Box 124, 22100 Lund, Sweden

P. Linse $(\bowtie)$

Division of Physical Chemistry, Department of Chemistry, Lund University, P.O. Box 124, 22100 Lund,

Sweden

e-mail: per.linse@fkem1.lu.se
} 
describing the dielectric behavior of a medium was considered solved by Kusalik [11, 12]. For that time, he modeled a very large system containing 4000 particles interacting via the so-called Stockmayer potential comprising a long-range dipole-dipole and a short-range Lennard-Jones term. It was for a long time assumed that the study of systems of that size was enough to guarantee that the dielectric behavior in general and the Kirkwood $g_{\mathrm{K}}$-factor was converged.

In a recent study of much larger systems involving up to 300000 particles [13], we showed that not even for these much larger systems could we expect to obtain a sizeindependent description of the dielectric properties of a high dielectric molecular fluid, as described by the distant-dependent Kirkwood $G_{\mathrm{k}}$-function. One way to access the convergence of the distance-dependent Kirkwood $G_{\mathrm{k}}$-function and the dielectric permittivity would be to systematically investigate the mechanisms behind the process. This has to the best of our knowledge never been done, but in the following sections we will try to shed some light on this problem by the use of Monte Carlo simulations of a spherically confined dipolar fluid surrounded by a dielectric medium. Before presenting this model and the obtained results, it is appropriate with a short theoretical discussion.

\section{Theoretical Considerations}

Kirkwood [5] suggested that the degree of dipolar order in a sphere of volume $V$ containing a dipolar fluid could be quantified by considering

$$
G_{\mathrm{k}}(r)=\frac{1}{\mu^{2}}\left\langle\sum_{j \subset V} \boldsymbol{\mu}_{1} \cdot \boldsymbol{\mu}_{j}\right\rangle
$$

where the sum runs over all dipoles in the sphere, which is centered at the position of the central dipole $\mu_{1}$ and possess the radius $r$. Thus, $G_{\mathrm{k}}(r)$ measures the degree of alignment of all dipoles in a sphere of radius $r$ with the direction of the central one. For random orientations, $G_{\mathrm{k}}(r)=1$ is obtained. The basic assumption by Kirkwood, as well as in this work, is that $G_{\mathrm{k}}(r)$ converges to a well-defined value, the $g_{\mathrm{K}}$-factor, for an infinite sphere in the thermodynamic limit.

In an ideal dielectric medium, (i) there is no length scale and (ii) Poissons equation predicts no dipolar order in the dielectric medium near a dipole in a cavity. This gives us reason to believe that the formation of dipolar order in a molecular dipolar fluid is linked to the only length scale present in the system, viz. the particle size as measured by either the range of the long-range dipole-dipole or the short-range interaction. Thus, it seems natural to associate the formation of the dipolar order, as, e.g., expressed by $G_{\mathrm{k}}(r)$, to the inhomogeneous molecular (i) positional and (ii) orientational order (collectively referred to as the packing) in the molecular fluid. We shall consequently modify the packing and investigate in what way this influences $G_{\mathrm{k}}(r)$.

The noncentral dipole-dipole interaction is the origin of the inhomogeneous orientational order in dipolar fluids. For example, the interaction in the direction of the dipole is stronger than that in the direction perpendicular to the dipole. Consequently, given two dipolar particles being at short separation, it is more likely to find them with coaxial dipolar axes than with perpendicular ones. This type of correlation is conventionally monitored by the angle $\theta$ between the direction of the dipole of one of the two dipolar particles and the vector between the dipolar particles. For each pair of particles, there are two angles of this type (see Fig. 1). 
Fig. 1 (Left) Illustration of a molecular fluid confined in a sphere with radius $R_{\mathrm{HS}}$ concentrically placed in a spherical cavity with radius $R_{\varepsilon}$ in a dielectric medium with the relative permittivity $\varepsilon$, and (right) the two angles $\theta_{1}$ and $\theta_{2}$ formed by the interparticle vector and the dipole directions of two dipolar particles

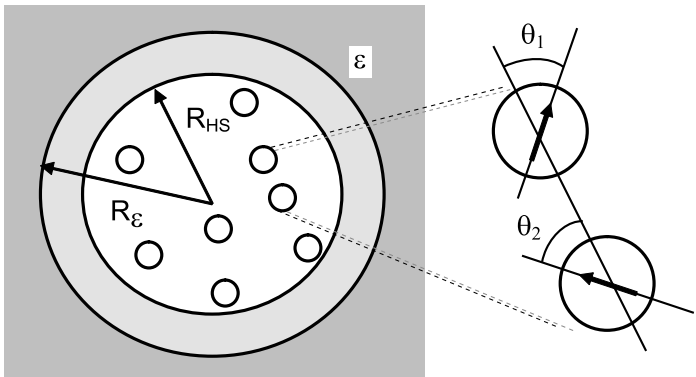

Our goal is to manipulate the molecular packing without changing the nature of the dipole-dipole interaction. For that purpose we have designed a penalty function $U_{\text {penal }}(r, \theta)$ consisting of two terms according to

$$
U_{\text {penal }}(r, \theta)=\lambda_{\mathrm{r}} U_{\mathrm{r}}(r)+\lambda_{\theta} U_{\theta}(r)\left[\frac{1}{2}\left(3 \cos ^{2} \theta_{1}-1\right)+\frac{1}{2}\left(3 \cos ^{2} \theta_{2}-1\right)\right]
$$

The first term $U_{\mathrm{r}}$ depends only on the distance $r$ between the particles and has the goal to establish a uniform radial distribution function $g(r)$. The second term depends both on $r$ and the angles $\theta_{1}$ and $\theta_{2}$ and its purpose is make $\left\langle 3 \cos ^{2} \theta\right\rangle$ equal to unity-the value of a uniform orientation distribution. As the orientational inhomogeneity depends on $r$, an $r$-dependent prefactor $U_{\theta}(r)$ is needed. For conveniences, we have also introduced the coupling parameters $\lambda_{\mathrm{r}}$ and $\lambda_{\theta}$ in (2). Thus, $\lambda_{\mathrm{r}}=\lambda_{\theta}=0$ represents an unperturbed (here Stockmayer) fluid, whereas $\lambda_{\mathrm{r}}=\lambda_{\theta}=1$ a perturbed fluid with ideally constant radial distribution function and constant orientational distribution. We introduce separate coupling parameters of the two terms of the penalty function to enable a study the effect of the radial and orientational structure separately.

If the molecular packing were responsible for the nonunity of the distance-dependent Kirkwood function $G_{\mathrm{k}}(r)$, we would expect that $G_{\mathrm{k}}(r)$ observed when the penalty function is applied would be smaller than for a nonpenalized system. Finally, note that we are not optimizing the penalty functions to minimize $G_{\mathrm{k}}(r)$ but to decrease the inhomogeneity of the fluid. Consequently, it is interesting to observe not only how $G_{\mathrm{k}}(r)$ is influenced when $\lambda$ is varied from 0 to 1 but also for values outside this interval.

\section{Model and Methods}

In this work, a spherically confined fluid composed of 1200 dipolar particles is studied using Monte Carlo simulations. The fluid is confined by an external hard-sphere potential with the radius $R_{\mathrm{HS}}=19.5 \AA$, which in turn is embedded in an outer spherical cavity with the radius $R_{\varepsilon}=20.7 \AA$. Moreover, the spherical cavity is surrounded by a dielectric medium with the relative permittivity $\varepsilon=130$ (see Fig. 1). The reaction field originating from the polarized dielectric surroundings acting on the molecular fluid is included in the model by using an image approximation as suggested by Friedman [14].

As previously [13], the dipole particles are specified by (i) the Lennard-Jones size parameter $\sigma=2.8863 \AA$ and interaction parameter $\varepsilon=1.97023 \mathrm{~kJ} / \mathrm{mol}$ and (ii) the dipole moment $\mu=1.651 \mathrm{D}$, whereas the system temperature $T=315.8 \mathrm{~K}$ is used. The analysis of the simulated system is based on the most central particle in the system to reduce the influence of the spherical boundary. 
Fig. 2 Illustration of the three paths employed in the $\left(\lambda_{\mathrm{r}}, \lambda_{\theta}\right)$-space describing the increase of the applied penalty starting from the no-penalty case $\left(\lambda_{\mathrm{r}}=\lambda_{\theta}=0\right)$ to the penalty minimizing the radial and orientational inhomogeneities $\left(\lambda_{\mathrm{r}}=\lambda_{\theta}=1\right)$

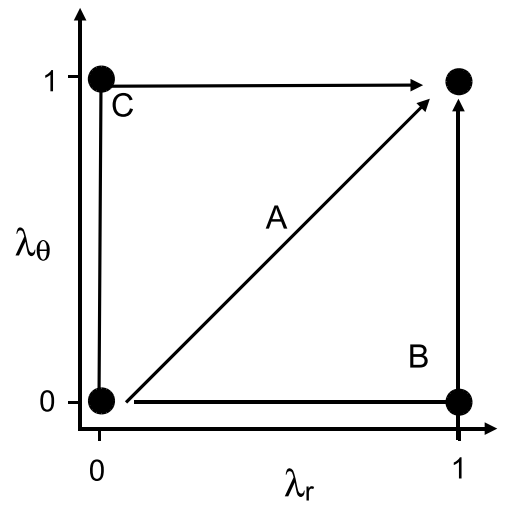

To investigate the effect of the packing of the particles on the distance-dependent Kirkwood function $G_{\mathrm{k}}(r)$, and thus on the dielectric permittivity, we have performed three sets of simulations where the weights of the two terms of the penalty function have systematically been varied. The sets comprised three paths from an unperturbed fluid $\left(\lambda_{\mathrm{r}}=\lambda_{\theta}=0\right)$ to a perturbed fluid with minimal structure $\left(\lambda_{\mathrm{r}}=\lambda_{\theta}=1\right)$. The paths were (A) $\lambda_{\mathrm{r}}=\lambda_{\theta}=\lambda$, (B) increase of $\lambda_{\mathrm{r}}$ from 0 to 1 followed by an increase of $\lambda_{\theta}$ from 0 to 1 , and (C) increase of $\lambda_{\theta}$ from 0 to 1 followed by an increase of $\lambda_{\mathrm{r}}$ from 0 to 1 (see Fig. 2). The case $\lambda_{\mathrm{r}}=\lambda_{\theta}=\lambda$, $\lambda<0$ has also been used to investigating the effect of increasing the structure of the spherically confined particles and $\lambda>1$ to generate structures with distinctly different characteristic than the unperturbed system. The simulations involved between $1 \cdot 10^{6}$ and $2 \cdot 10^{6}$ trial moves per particle, $1 \cdot 10^{6}$ for $0 \leq \lambda \leq 1$ and the longer simulations for $\lambda<0$ and $\lambda>1$, where worse statistics were obtained when the structure was enhanced.

Regarding the penalty function $U_{\text {penal }}(r, \theta)$ defined by (1), $U_{\mathrm{r}}(r)$ and $U_{\theta}(r)$ were determined using an iterative process. They were updated according to

$$
\begin{aligned}
& U_{\mathrm{r}}^{\text {new }}(r)=U_{\mathrm{r}}^{\text {old }}(r)-\gamma \exp \left(-s r^{2}\right) \ln \left[g^{\text {new }}(r)\right] \\
& U_{\theta}^{\text {new }}(r)=U_{\theta}^{\text {old }}(r)-\gamma \exp \left(-s r^{2}\right)\left[\frac{1}{2}\left\langle 3 \cos ^{2} \theta_{1}-1\right\rangle_{\text {new }}+\frac{1}{2}\left\langle 3 \cos ^{2} \theta_{2}-1\right\rangle_{\text {new }}\right]
\end{aligned}
$$

where the used radial distribution function $g^{\text {new }}(r)$ and the orientational average $\left\langle 3 \cos ^{2} \theta\right\rangle_{\text {new }}(r)$, involving the central particle obtained in a simulation using $U^{\text {old }}$ from the previous iteration, were used to construct a new estimate $U^{\text {new }}$ of the penalty function. Furthermore, (i) the radial factor $\exp \left(-s r^{2}\right)$ was invoked to make the penalty function short ranged [ $s$ was chosen to make $U_{\text {penal }}(r, \theta)$ negligible $\left(\approx 10^{-4} \mathrm{kT}\right)$ at $r=15 \AA$ ], (ii) the prefactor $\gamma=0.6$ was included to avoid the updating procedure to become oscillatory, (iii) $U_{\mathrm{r}}^{\text {old }}(r)=U_{\theta}^{\text {old }}=0$ were used in the first iteration, and (iv) at convergence, in principle, $U_{\mathrm{r}}^{\text {new }}(r)=U_{\mathrm{r}}^{\text {old }}(r)$ and $U_{\theta}^{\text {new }}(r)=U_{\theta}^{\text {old }}(r)$. However, as convergence implies $g(r)=1$ for all $r$, including $r<\sigma$, and as we desired to retain the excluded volume effect of the particles, the iteration was stopped before the onset $g(r)>0$ was shifted more than $0.2 \AA$, even if $g(r>\sigma)$ and the orientation distribution were not fully converged. In retrospect, this problem would have been avoided, if a hard-sphere short-range potential had been used. Nevertheless, the results below are sufficient to draw reliable conclusions about the origin of the distance-dependent Kirkwood function. 
Fig. 3 (Color online) Radial distribution function $g(r)$ and radial orientational-averaged distribution $\left\langle 3 \cos ^{2} \theta\right\rangle(r)$ obtained without $\left(\lambda_{\mathrm{r}}=\lambda_{\theta}=0\right)$ and with $\left(\lambda_{\mathrm{r}}=\lambda_{\theta}=1\right)$ penalty function applied. For better clarity $g(r)$ is shifted +0.4 in the $y$-direction

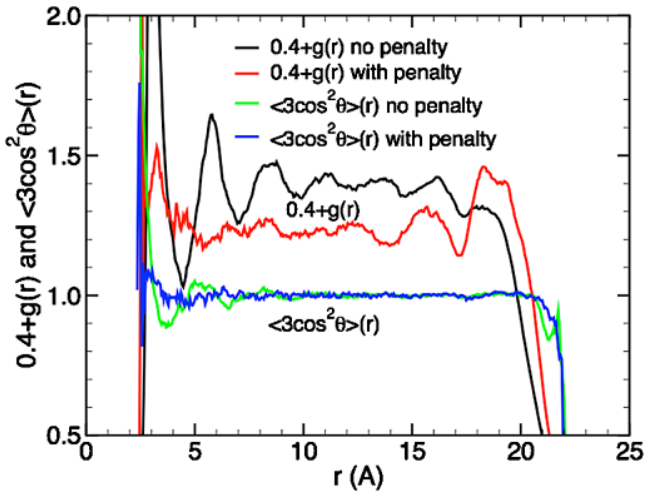

\section{Results and Discussions}

The radial distribution function $g(r)$ and the radial orientational average $\left\langle 3 \cos ^{2} \theta\right\rangle(r)$ around the most central particle in the confining sphere obtained without $\left(\lambda_{\mathrm{r}}=\lambda_{\theta}=0\right)$ and with $\left(\lambda_{\mathrm{r}}=\lambda_{\theta}=1\right)$ the penalty function applied are displayed in Fig. 3.

The unperturbed fluid displays a radial distribution function possessing (i) the conventional set of maxima with decreasing amplitude appearing at $r \approx n \sigma$, where $n$ is an integer, and (ii) an approach to unity at longer distance. Since the present simulations are performed in a sphere with a hard-wall potential, the structure at large $r$ (say $r>15 \AA$ ) becomes influenced by this wall and will here not be treated. Similarly, $\left\langle 3 \cos ^{2} \theta\right\rangle(r)$ deviates from unity at short distance where it also displays an oscillatory decaying behavior. A positive value implies a preferential location of the particles in the forward and backward dipole direction and a negative value a preferential location perpendicular to the dipole direction. The maxima of $\left\langle 3 \cos ^{2} \theta\right\rangle(r)$ appear roughly a quarter of a period before those of $g(r)$. The extreme points of $\left\langle 3 \cos ^{2} \theta\right\rangle(r)$ deviate from unity much less than those of $g(r)$ do. As we will see below, this deviation is however of large importance.

For the fluid with the penalty function applied, the radial distribution function becomes much smoother, though not completely smooth as already alluded to. Noticeable are the reminisces of the maximum of the unperturbed system at $r \approx \sigma$ and the rise of the radial distribution function appearing ca $0.2 \AA$ earlier than in the unperturbed system. Further iterations of the penalty function improve the smoothness of $g(r \approx \sigma)$ at the expense of further shrinkage of the excluded volume. Moreover, at intermediate distance the radial distribution function becomes smaller than unity due to an increased density of particles near the spherical boundary. Regarding $\left\langle 3 \cos ^{2} \theta\right\rangle(r)$, it becomes nearly completely uniform with the penalty function applied.

Figure 4 shows the radial term $U_{\mathrm{r}}(r)$ and the prefactor $U_{\theta}(r)$ of the orientational term of the penalty function. Both quantities display oscillations at short $r$ with a wavelength of $\approx \sigma$ and approach smoothly zero at increasing $r$ as constructed. At $r \approx \sigma$, (i) $U_{\mathrm{r}}(r)$ becomes large to suppress the maximum in the radial distribution function at that $r$ and (ii) $U_{\theta}(r)$ becomes positive to suppress the dipolar alignment at this distance (cf. Fig. 3). At shorter distance, $U_{\mathrm{r}}(r)$ becomes rapidly and strongly negative to match the steep rise of the Lennard-Jones potential.

The distance-dependent Kirkwood function $G_{\mathrm{k}}(r)$ obtained from series A with $\lambda_{\mathrm{r}}=\lambda_{\theta}=$ $\lambda$ are presented in Fig. 5a. First, the unperturbed system $(\lambda=0)$ displays a $G_{\mathrm{k}}(r)$ that increases from unity at small $r$ in two steps to $G_{\mathrm{k}} \approx 4$ at $r \approx 2 \sigma$ and continues with a gentle 
Fig. 4 (Color online) Reduced radial term $U_{\mathrm{r}}(r) / k T$ and reduced prefactor $U_{\theta}(r) / k T$ of the orientational term of the penalty function versus the distance $r$

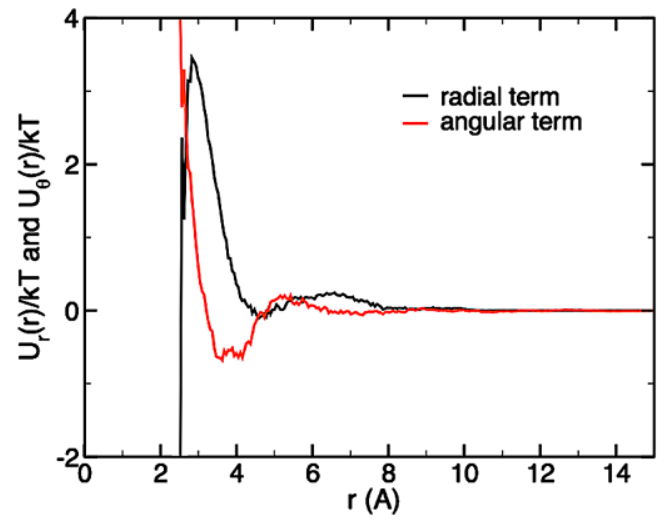

Table 1 Dipolar energy per particle (in kT) as a function of $\lambda_{\mathrm{r}}$ and $\lambda_{\theta}$

\begin{tabular}{llll}
\hline$\lambda_{\theta}$ & $\lambda_{\mathrm{r}}$ & & \\
\cline { 2 - 4 } & 0.0 & 0.5 & 1.0 \\
\hline 0.0 & -4.32 & -4.48 & -5.39 \\
0.5 & -3.74 & -3.83 & -4.18 \\
1.0 & -3.52 & -3.67 & -4.18 \\
\hline
\end{tabular}

rise to 5 at $r \approx 4 \sigma$. Thus, on the average, the dipoles of the particles in the first as well as in second shell of neighbors of the central particle are preferentially oriented parallel to the dipole of the central one. The preferential orientation of particles further away is weak.

Second, a strong dependence of $G_{\mathrm{k}}(r)$ on the magnitude of the penalty function applied through $\lambda$ is found. Positive $\lambda$ values decrease $G_{\mathrm{k}}(r)$, whereas negative $\lambda$ values increase this quantity. (i) For unit value of $\lambda$, there is practically no dipolar structure left as analyzed by $G_{\mathrm{k}}(r)$, consistent with nearly annealed radial and orientational structure. (ii) For $\lambda>1$, $G_{\mathrm{k}}(r)$ initially decreases below 1 and then starts to grow slowly but it always remains small. (iii) Furthermore, with a negative coupling parameter $G_{\mathrm{k}}(r)$ displays a stronger rise at distances corresponding to the first two shells and the increase continues steadily at even larger distances from the central particle. The overall main increase of $G_{\mathrm{k}}(r)$ appears already at $\lambda=-0.25$ and that a more negative $\lambda$ induces only a minor effect on the $G_{\mathrm{k}}(r)$. Thus, we have demonstrated that by suppressing the radial and orientational molecular inhomogeneities of the dipolar fluid, we reduce the dipolar correlations and hence the dielectric response of the fluid and a opposite response is achieved when the inhomogeneities are enforced.

It is also worth mentioning that (i) the systems obtained using $\lambda>1$ exhibit maxima in $g(r)$ and $\left\langle 3 \cos ^{2} \theta\right\rangle(r)$, at which the unperturbed system shows minima and vice versa, and that systems generated with $\lambda<0$ show an increase structure as measured by $g(r)$ and $\left\langle 3 \cos ^{2} \theta\right\rangle(r)$ in comparison to the unperturbed system (data not shown).

We will continue our analyses by considering if $G_{\mathrm{k}}(r)$ depends on the radial or orientational correlations or if perhaps on both. An obvious way to perform this is to study the effect of varying the magnitude of the radial and orientational term of the penalty function separately. Figure $5 \mathrm{~b}$ displays the effect of first varying $\lambda_{\mathrm{r}}$ and then $\lambda_{\theta}$ along bath $\mathrm{B}$ given by Fig. 2, and Fig. 5c shows the effect of first varying $\lambda_{\theta}$ followed by varying $\lambda_{\mathrm{r}}$ as in path $\mathrm{C}$ of Fig. 2. In panels $\mathrm{b}$ and $\mathrm{c}$ of Fig. 5, the top curves give $G_{\mathrm{k}}(r)$ without $\left(\lambda_{\mathrm{r}}=\lambda_{\theta}=0\right)$ and the 
Fig. 5 (Color online)

Distance-dependent Kirkwood

function $G_{\mathrm{k}}(r)$ versus $r$ at

various degree of penalty applied

through the coupling parameters

$\lambda_{\mathrm{r}}$ and $\lambda_{\theta}$ [see (2)] for various

combinations of $\lambda_{\mathrm{r}}$ and $\lambda_{\theta}$ as predescribed by (a) path A,

(b) path $\mathrm{B}$, and (c) path $\mathrm{C}$ shown in Fig. 2. The values of coupling parameters (a) $\lambda$ and (b and c) $\lambda_{\mathrm{r}}$ and $\lambda_{\theta}$ used are given in the curve captions appearing in the panels
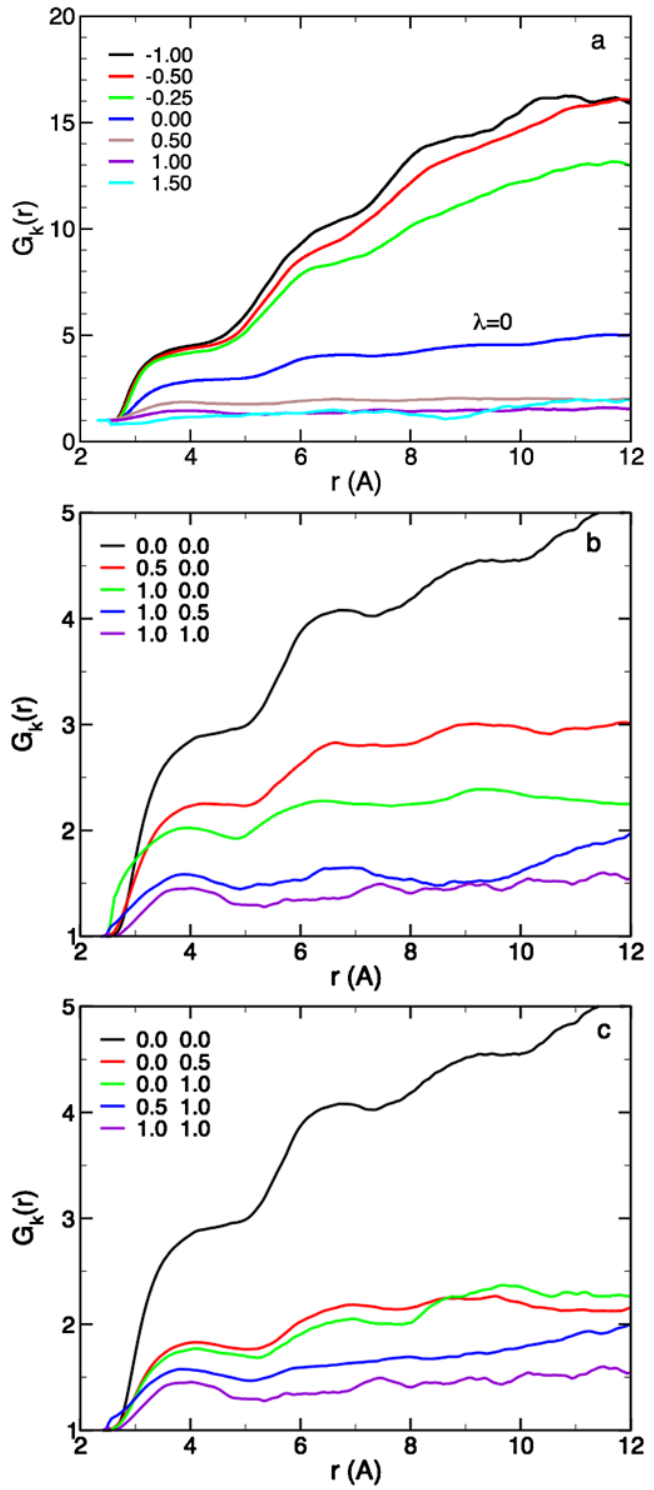

bottom ones with penalty $\left(\lambda_{\mathrm{r}}=\lambda_{\theta}=1\right)$ applied. The data shows that $G_{\mathrm{k}}(r)$ decays monotonically along both paths and though the largest response appears when applying the orientational penalty, it is clear that both the radial and orientational term of the penalty function are of importance. Hence, an important message is that the high values of the distancedependent Kirkwood function in a simple polar fluid is intimately related to both the radial and orientational packing of the particles.

The change of the packing of the particles will of course affect the way the dipoles are interacting, and one way to monitor this is to study the average dipole-dipole interaction. Table 1 provides average dipolar energies for different values of $\lambda_{\mathrm{r}}$ and $\lambda_{\theta}$. It is clear that the use of the radial term of the penalty function strengthens the dipole-dipole interaction. The 
main explanation is that the chosen radial penalty function actually facilitates the dipoles to come closer, as discussed above. The effect of the orientational term of the penalty function is to weaken the dipole-dipole interaction. The main explanation for this is that the linear and attractive alignment of dipoles at short distances, which corresponds to the most favorable arrangement, is strongly penalized.

\section{Conclusions}

This work shows that it is possible to control the dipolar structure in a fluid by manipulating the radial and orientational structure of the dipolar particles by employing a short-range penalty function. We have shown that the long-range dipolar order is intimately coupled to the radial and orientational inhomogeneity of the dipolar fluid. In particular, we note that the dipolar structure is enhanced by stronger radial packing and decreased when suppressing the orientational inhomogeneity. In this work, a penalty function controlled the radial packing and orientational inhomogeneity, and in a following contribution [15] we systematically investigate how the same objective can be achieved by modifying the shape of the particles and by introducing higher electrostatic moments of the particles.

Acknowledgements Financial support by the Swedish Research Council (VR) through the Linnaeus grant Organizing Molecular Matter (OMM) center of excellent (239-2009-6794) and through individual grants to GK (621-2007-5009) and PL (621-2007-5251) is gratefully acknowledged.

Open Access This article is distributed under the terms of the Creative Commons Attribution Noncommercial License which permits any noncommercial use, distribution, and reproduction in any medium, provided the original author(s) and source are credited.

\section{References}

1. Clausius, R.: Die Mechanische Wärmetheorie, vol. II, p. 62. Braunschweig (1879)

2. Mossotti, O.F.: Bibl. Univ. Modena 6, 193 (1847)

3. Langvin, P.: J. Phys. 4, 678 (1905)

4. Onsager, L.: J. Am. Chem. Soc. 58, 1486 (1936)

5. Kirkwood, J.G.: J. Chem. Phys. 7, 911 (1939)

6. Nienhuis, G., Deutch, J.M.: J. Chem. Phys. 56, 1819 (1972)

7. Wertheim, M.S.: Ann. Rev. Phys. Chem. 30, 471 (1979)

8. Ladd, A.J.C.: Mol. Phys. 36, 463 (1978)

9. Adams, D.J., McDonald, I.R.: Mol. Phys. 32, 931 (1976)

10. Neumann, M.: Mol. Phys. 50, 841 (1983)

11. Kusalik, P.G.: J. Chem. Phys. 93, 3520 (1990)

12. Kusalik, P.G.: Mol. Phys. 73, 1349 (1991)

13. Stenhammar, J., Linse, P., Karlström, G.: J. Chem. Phys. 131, 164507 (2009)

14. Friedman, H.L.: Mol. Phys. 29, 1533 (1975)

15. Linse, P., Karlström, G.: J. Stat. Phys., doi:10.1007/s10955-011-0352-2 